\title{
Viscosity Measurements of Liquid Toluene at Low Temperatures Using a Dual Vibrating-Wire Technique
}

\author{
F. J. P. Caetano, ${ }^{1,2}$ J. L. Correia da Mata, ${ }^{1,3}$ J. M. N. A. Fareleira, ${ }^{1,4}$ \\ C. M. B. P. Oliveira, ${ }^{1,2}$ and W. A. Wakeham ${ }^{5}$
}

Received April 28, 2003

\begin{abstract}
A recently developed dual vibrating-wire technique has been used to perform viscosity measurements of liquid toluene in the temperature range $213 \mathrm{~K} \leqslant$ $T \leqslant 298 \mathrm{~K}$, and at pressures up to approximately $20 \mathrm{MPa}$. The results were obtained by operating the vibrating-wire sensor in both forced and free decay modes. The estimated precision of the viscosity measurements, in either mode of operation, is $\pm 0.5 \%$, for temperatures above or equal to $273 \mathrm{~K}$, increasing with decreasing temperature up to $\pm 1 \%$ at $213 \mathrm{~K}$. The corresponding overall uncertainty is estimated to be within $\pm 1 \%$ and $\pm 1.5 \%$, respectively.
\end{abstract}

KEY WORDS: dual vibrating-wire technique; low temperature; toluene; viscosity.

\section{INTRODUCTION}

We have previously described a vibrating-wire instrument, operated in a forced mode, which has been used for the simultaneous measurement of the density and the viscosity of fluids, over wide ranges of temperature and pressure [1-4]. Recently [5], we have described some preliminary tests of a novel instrument, showing that it is possible to perform mutually consistent viscosity measurements by operating a vibrating-wire sensor both in

\footnotetext{
${ }^{1}$ Centro de Química Estrutural, Instituto Superior Técnico, Universidade Técnica de Lisboa, Av. Rovisco Pais, 1049-001 Lisbon, Portugal.

${ }^{2}$ Universidade Aberta, Rua da Escola Politécnica, 147, 1269-001 Lisbon, Portugal.

${ }^{3}$ Academia Militar, Paço da Rainha, 29, 1150-244 Lisbon, Portugal.

${ }^{4}$ To whom correspondence should be addressed. E-mail: j.fareleira@ist.utl.pt

${ }^{5}$ University of Southampton, Highfield, Southampton SO17 1BJ, United Kingdom.
} 
forced and free decay modes. The present work reports viscosity measurements of toluene, obtained by the latter instrument using both modes of operation, at low temperatures and pressures up to $20 \mathrm{MPa}$. New electronic equipment has been purposely built that enables the monitoring of either the frequency or the transient responses of the vibrating-wire sensor. Both modes of operation are described by an exact theory, so that the viscosity can be obtained from an analysis of the resonance curve, or from the time response of the sensor $[5,6]$.

The consistency of the two approaches, under the same working conditions, as well as the quality of the measuring system, have been assessed during the measurements. It is concluded that the new equipment improves the forced mode of operation, adds new flexibility to the vibrating-wire method, and provides further evidence that the instrument is working according to its theoretical description.

\section{THEORETICAL MODEL}

The theoretical model for the vibrating-wire viscometer developed by Retsina et al. $[7,8]$ is based on the characteristics of the oscillation of a solid body surrounded by a fluid. The theoretical model for the forced mode of operation has been published by Pádua et al. [9], and, for the free decay mode of operation, the model equations are essentially analogous to the ones previously described by Oliveira [10] and Assael et al. [11]. Hence, only a brief description of the working equations for both modes is given here.

For the forced mode, the wire's velocity resonant frequency, $\omega_{r}$, is the solution of the equation $[7,9]$ :

$$
\frac{d}{d \tilde{\omega}}\left\{\tilde{\omega}^{2}\left(\beta^{\prime}+2 \Delta_{0}\right)^{2}+\left[\tilde{\omega}(1+\beta)-\frac{\tilde{\omega}_{B}^{2}}{\tilde{\omega}}\right]^{2}\right\}=0
$$

and the half-width, of a resonance curve, $\Delta \omega=\omega_{+}-\omega_{-}$, is related to the cell parameters through the equation:

$$
\begin{aligned}
& \frac{\tilde{\omega}_{ \pm}\left(\beta^{\prime}+2 \Delta_{0}\right)}{\tilde{\omega}_{ \pm}^{2}\left(\beta^{\prime}+2 \Delta_{0}\right)^{2}+\left[\tilde{\omega}_{ \pm}(1+\beta)-\frac{\tilde{\omega}_{B}^{2}}{\tilde{\omega}_{ \pm}}\right]^{2}} \\
& =\frac{1}{2} \frac{\tilde{\omega}_{r}^{2}\left(\beta^{\prime}+2 \Delta_{0}\right)}{\tilde{\omega}_{r}^{2}\left(\beta^{\prime}+2 \Delta_{0}\right)^{2}+\left[\tilde{\omega}_{r}(1+\beta)-\frac{\tilde{\omega}_{B}^{2}}{\tilde{\omega}_{r}}\right]^{2}}
\end{aligned}
$$


The tilde above a symbol indicates a corresponding dimensionless quantity, as defined in [7], such that the relation between a dimensionless frequency and the corresponding angular frequency, $\omega$, is given by:

$$
\tilde{\omega}=\omega\left(\frac{4 \rho_{s} L^{4}}{E R^{2}}\right)^{1 / 2}
$$

where $\rho_{s}$ is the wire material density, $E$ is its Young's modulus, $L$ is the halflength of the wire, and $R$ is its radius.

In the free decay mode, the wire's oscillation frequency, $\omega$, and the rate of decay, $\Delta$, are described in terms of the cell parameters, by Eqs. (4) and $(5)[8,10,11]$ :

$$
\begin{aligned}
\Delta & =\frac{\beta^{\prime}+2 \Delta_{0}}{2(\beta+1)} \\
\omega^{2} & =\frac{\left(1+\Delta_{0}^{2}\right) \omega_{B}^{2}}{(1+\beta)\left(1-\Delta^{2}\right)+\Delta\left(\beta^{\prime}+2 \Delta_{0}\right)}
\end{aligned}
$$

In the above equations, $\Delta_{0}$ is the internal damping of the wire and $\omega_{B}$ represents a resonant frequency of the wire, in the hypothetical situation where the wire is under vacuum and the weights tensioning it vertically are immersed in the fluid.

The oscillation characteristics of the vibrating-wire sensor, both in forced and free decay modes of operation, are dependent on the density, $\rho$, and viscosity, $\eta$, of the surrounding fluid, as well as on $\rho_{s}$, through the functions:

$$
\beta=\left(\rho / \rho_{s}\right) k \quad \text { and } \quad \beta^{\prime}=\left(\rho / \rho_{s}\right) k^{\prime}
$$

where, in the forced mode:

$$
\begin{gathered}
k=-1+2 \mathfrak{I} m(\mathscr{A}) \quad \text { and } \quad k^{\prime}=2 \Re e(\mathscr{A}) \\
\text { with } \quad \mathscr{A}=i\left[1+\frac{2 K_{1}(\sqrt{i \Omega})}{\sqrt{i \Omega} K_{0}(\sqrt{i \Omega})}\right]
\end{gathered}
$$

In the free decay mode a corresponding set of equations applies:

$$
\begin{gathered}
k=-1+2 \mathfrak{I} m(\mathscr{A}) \quad \text { and } \quad k^{\prime}=2 \mathfrak{R} e(\mathscr{A})+2 \Delta \mathfrak{I} m(\mathscr{A}) \\
\text { with } \quad \mathscr{A}=(i-\Delta)\left(1+\frac{2 K_{1}(\sqrt{(i-\Delta) \Omega})}{\sqrt{(i-\Delta) \Omega} K_{0}(\sqrt{(i-\Delta) \Omega})}\right)
\end{gathered}
$$


$K_{0}$ and $K_{1}$ are modified complex argument Bessel functions, and $\Omega$ is a dimensionless frequency related to the Reynolds number for the fluid motion around the wire:

$$
\Omega=\frac{\rho \omega R^{2}}{\eta}
$$

\section{EXPERIMENTAL SETUP}

The current viscosity measurements of toluene at temperatures $213 \mathrm{~K} \leqslant T \leqslant 298 \mathrm{~K}$, and pressures up to $20 \mathrm{MPa}$, have been carried out with a vibrating-wire viscometer described in detail elsewhere $[5,6]$.

The vibrating-wire sensor consists of a tungsten wire with a nominal diameter of $100 \mu \mathrm{m}$. The wire is suspended from a stainless steel chuck supported from the cap of a pressure vessel. At its lower end an assembly composed of two weights, through a pulley system, tensions the wire vertically. The main aim of this assembly is to inhibit any motion of the wire lower end, as discussed previously [11].

The measuring cell was placed inside a stainless steel pressure vessel. For the present range of temperature the thermostatic system was based on a circulation cryostat (Colora KT 190S), using ethanol as the circulating fluid. The temperature stability during a typical run was within $\pm 0.005 \mathrm{~K}$. Temperature measurements were performed using a platinum resistance thermometer with an uncertainty of $\pm 0.01 \mathrm{~K}$ (ITS-90). Pressure readings were carried out using a pressure transducer, supplied by Setra Systems Inc, with an uncertainty of $\pm 0.13 \%$ (FS).

\subsection{Electrical Measuring Device/Signal Detection}

The new electronic equipment, which is described in detail elsewhere [6], was purposely developed to enable the use of both forced and free decay modes of operation. In the forced mode a resonance curve is obtained by measuring the impedance of the vibrating-wire sensor, using a four-wire technique, over an adequate frequency range about the resonant frequency. The forced mode oscillations are induced by passing a sinusoidal current through the wire, placed in the gap of a magnetic circuit. The response of the wire can thus be interpreted as a complex impedance, $\bar{Z}$,

$$
\bar{Z}=R_{s}+i \omega \mathscr{L}_{s}+\frac{1}{G \omega+i \omega C+\frac{1}{i \omega \mathscr{L}_{p}}}
$$

of an equivalent electrical circuit, as shown in Fig. 1. 


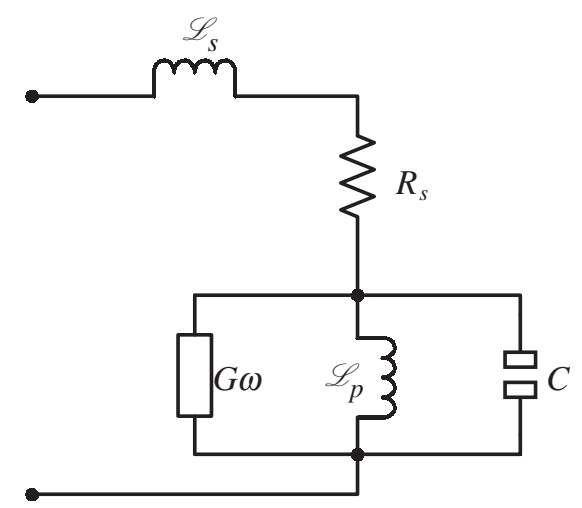

Fig. 1. Complete equivalent electrical circuit model of the oscillating wire in forced mode.

The three parallel elements: the capacitor $C$, the inductor $\mathscr{L}_{p}$, and the frequency dependent conductance $G \omega$, describe the resonance characteristics of the wire. The series resistor, $R_{s}$, models the circuit resistance, and the inductance, $\mathscr{L}_{s}$, is caused mainly by the high-pressure feed-through. In a typical experiment these parameters are obtained by fitting the experimental data to the modulus of Eq. (10).

In the free decay mode of operation a symmetrical current pulse is employed to deflect the wire from its rest position. The subsequent decaying oscillations are detected by the induced voltage at the wire ends, in an electro-magnetic coupling device, with a 12-bit A/D converter at a rate of $10^{5}$ samples per second. The corresponding transient decay signal is expected to conform to the following equation:

$$
V=A e^{-\Delta \omega t} \sin (\omega t+\phi)
$$

where the measured voltage, $V$, and the time interval, $t$, are the experimental parameters. The amplitude $A$, the logarithmic decrement $\Delta$, the frequency of oscillation $\omega$, and the phase shift $\phi$, are obtained through nonlinear fitting.

\subsection{Cell Parameters}

The working equations (Eqs. (1)-(5)) incorporate several parameters that are independent of the fluid, namely, $\Delta_{0}, L, R$, and $\rho_{\mathrm{s}}$. Once these and the density of the fluid sample are known, the viscosity of the fluid can be obtained from the resonance frequency and the width of the resonance curve - forced mode - or from the measured frequency and the decay of the motion-free decay mode. 
Table I. Cell Parameters at $298 \mathrm{~K}$

\begin{tabular}{lcc}
\hline Length of the wire & $2 L$ & $0.04077 \mathrm{~m}$ \\
\hline Radius of the wire & $R$ & $48.965 \times 10^{-6} \mathrm{~m}$ \\
\hline Density of the wire [12] & $\rho_{s}$ & $19230 \mathrm{~kg} \cdot \mathrm{m}^{-3}$ \\
\hline
\end{tabular}

The length of the wire is measured, using a cathetometer, with an uncertainty of $\pm 0.05 \%$, and the density of the wire is taken from the literature [12]. The accuracy target in the viscosity measurements is incompatible with a direct measurement of the wire radius. Therefore, this parameter is advantageously determined in a single experimental run, under thermodynamic conditions where the viscosity of a reference fluid is well known. Hence, a single experimental run in the forced mode, with toluene as a reference fluid was performed near $298 \mathrm{~K}$ and $0.1 \mathrm{MPa}$ in order to obtain an effective average radius for the wire sensor. The viscosity and density reference data for toluene were taken from Gonçalves et al. [13] and Kashiwagi et al. [14], respectively.

The actual cell parameters for the present setup, at $298 \mathrm{~K}$, are shown in Table I. Given that the current measurements are concerned solely with viscosity, the only calibration parameter is the wire radius, which is essentially determined from the viscosity of the calibration fluid.

\section{EXPERIMENTAL RESULTS}

\subsection{Sample}

The sample of toluene (Sigma Aldrich, HPLC grade, with a nominal purity of $99.8 \%$ ) was dried using molecular sieves (Riedel-deHaën, $0.4 \mathrm{~nm}$ ), over a long period of exposure. The final water content was less than $10 \mathrm{ppm}$ as shown by a Karl-Fischer titration analysis. The toluene was introduced into the vessel, under vacuum.

\subsection{Density Data}

A new correlation for the density of toluene has been developed by Assael et al. [15], based on experimental data from 198 to $380 \mathrm{~K}$ and up to $280 \mathrm{MPa}$. This Tait-type correlation, with a claimed uncertainty of $\pm 0.1 \%$, within $95 \%$ confidence limits, has been used in the present work in order to provide the density of liquid toluene over the temperature and pressure ranges of the viscosity measurements. It should be noted that a previously 
performed sensitivity analysis has indicated that an uncertainty of $\pm 0.1 \%$ in the density yields an uncertainty of approximately $\pm 0.1 \%$ in the viscosity for the present measurements.

\subsection{Experimental Measurements}

The estimated precision of the present viscosity measurements, either in forced or free decay modes of operation, is $\pm 0.5 \%$ for temperatures above $273 \mathrm{~K}$, increasing with decreasing temperature up to $1 \%$ at $213 \mathrm{~K}$. The mutual consistency of the results obtained using the two modes was verified for the entire temperature range at several pressures, as shown in Fig. 2. It should be noted that each point represents the average of two or three experimental values. It can be seen that the differences between the results obtained by the two modes are mostly within $\pm 0.4 \%$, which adequately confirms the internal consistency of the two modes of operation for viscosity measurements. The corresponding overall uncertainty is estimated to be within $\pm 1 \%$ and $\pm 1.5 \%$, at temperatures above, and below, $273 \mathrm{~K}$, respectively.

In Table II the results of the present measurements for the viscosity of toluene as a function of pressure are shown, together with the corresponding density values, for the temperature range of 213 to $298 \mathrm{~K}$, and pressures up to approximately $20 \mathrm{MPa}$. These data are the result of an average of two or three experimental values obtained in sets of measurements performed both in increasing and decreasing cycles of pressure. No hysteresis

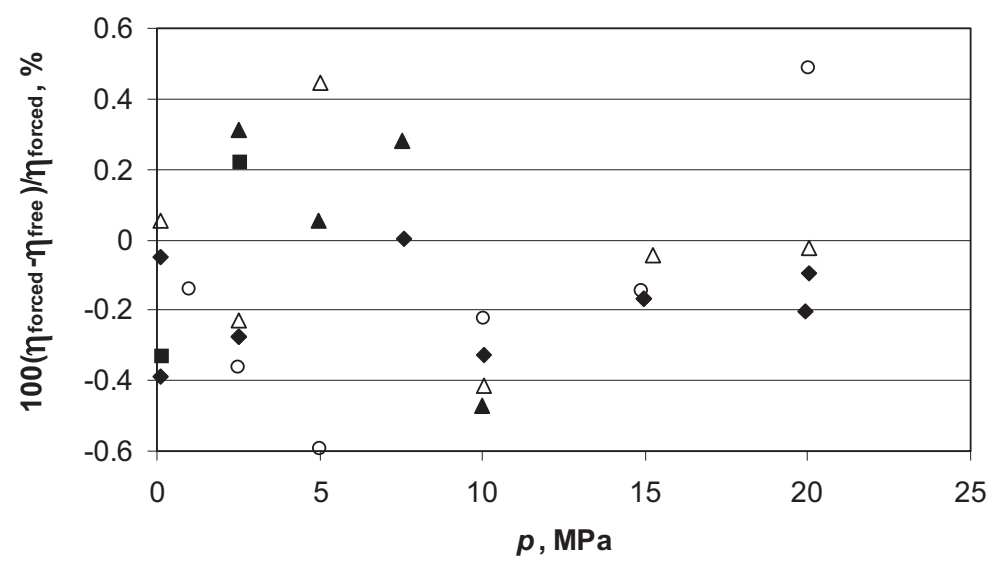

Fig. 2. Differences of the viscosity measurements for toluene, using forced and free decay modes of operation: $(\bullet) 298.31 \mathrm{~K}$, (⿴) $273.81 \mathrm{~K},(\boldsymbol{\Delta}) 248.47 \mathrm{~K}$, (○) $222.57 \mathrm{~K},(\triangle) 213.45 \mathrm{~K}$. 
Table II. Experimental Viscosity Measurements of Toluene, as a Function of Temperature and Pressure. (The density data were evaluated according to the correlation equation given in [15])

\begin{tabular}{|c|c|c|c|c|c|}
\hline$p(\mathrm{MPa})$ & $\rho\left(\mathrm{kg} \cdot \mathrm{m}^{-3}\right)$ & $\eta(\mathrm{mPa} \cdot \mathrm{s})$ & $p(\mathrm{MPa})$ & $\rho\left(\mathrm{kg} \cdot \mathrm{m}^{-3}\right)$ & $\eta(\mathrm{mPa} \cdot \mathrm{s})$ \\
\hline \multicolumn{3}{|c|}{$T=298.31 \mathrm{~K}$} & \multicolumn{3}{|c|}{$T=273.15 \mathrm{~K}$} \\
\hline 0.12 & 862.4 & 0.5543 & 0.13 & 885.0 & 0.7637 \\
\hline 0.13 & 862.4 & $0.5550^{a}$ & 0.14 & 885.0 & 0.7627 \\
\hline 0.13 & 862.4 & 0.5539 & 0.14 & 885.0 & $0.7652^{a}$ \\
\hline 2.57 & 864.3 & $0.5637^{a}$ & 2.51 & 886.6 & 0.7790 \\
\hline 2.57 & 864.3 & 0.5684 & 2.54 & 886.6 & $0.7777^{a}$ \\
\hline 4.96 & 866.1 & 0.5743 & 2.54 & 886.6 & 0.7794 \\
\hline 4.97 & 866.1 & $0.5724^{a}$ & 5.00 & 888.3 & 0.7933 \\
\hline 4.97 & 866.1 & 0.5753 & 5.07 & 888.3 & 0.7936 \\
\hline 5.04 & 866.1 & 0.5756 & 9.98 & 891.5 & 0.8246 \\
\hline 7.60 & 868.0 & $0.5867^{a}$ & 10.03 & 891.5 & 0.8265 \\
\hline 7.60 & 868.0 & 0.5865 & & & \\
\hline 9.12 & 869.2 & 0.5940 & & & \\
\hline 10.01 & 869.8 & 0.5979 & & & \\
\hline 10.05 & 869.8 & $0.6006^{a}$ & & & \\
\hline \multirow[t]{2}{*}{10.05} & 869.8 & 0.5986 & & & \\
\hline & \multicolumn{2}{|l|}{$T=248.47 \mathrm{~K}$} & \multicolumn{3}{|c|}{$T=222.57 \mathrm{~K}$} \\
\hline 0.12 & 908.4 & 1.178 & 0.11 & 932.5 & 2.099 \\
\hline 0.13 & 908.4 & 1.178 & 1.00 & 932.9 & 2.115 \\
\hline 0.13 & 908.4 & $1.185^{a}$ & 1.00 & 932.9 & $2.118^{a}$ \\
\hline 2.49 & 909.8 & 1.200 & 2.52 & 933.7 & 2.148 \\
\hline 2.49 & 909.8 & $1.197^{a}$ & 2.52 & 933.7 & $2.155^{a}$ \\
\hline 4.96 & 911.2 & 1.226 & 4.97 & 934.9 & 2.195 \\
\hline 4.96 & 911.2 & $1.225^{a}$ & 5.00 & 935.0 & $2.217^{a}$ \\
\hline 7.52 & 912.7 & 1.252 & 5.00 & 935.0 & 2.204 \\
\hline 7.52 & 912.7 & $1.248^{a}$ & 7.52 & 936.2 & $2.266^{a}$ \\
\hline 9.98 & 914.1 & 1.278 & 7.52 & 936.2 & 2.235 \\
\hline 10.00 & 914.1 & 1.278 & 10.02 & 937.5 & 2.311 \\
\hline \multirow[t]{5}{*}{10.00} & 914.1 & $1.284^{a}$ & 10.02 & 937.5 & $2.316^{a}$ \\
\hline & & & 14.91 & 939.8 & $2.429^{a}$ \\
\hline & & & 14.91 & 939.8 & 2.426 \\
\hline & & & 20.04 & 942.3 & $2.533^{a}$ \\
\hline & & & 20.04 & 942.3 & 2.545 \\
\hline
\end{tabular}

${ }^{a}$ Values obtained by using free decay mode. 
Table II. (Continued)

\begin{tabular}{|c|c|c|c|c|c|}
\hline$p(\mathrm{MPa})$ & $\rho\left(\mathrm{kg} \cdot \mathrm{m}^{-3}\right)$ & $\eta(\mathrm{mPa} \cdot \mathrm{s})$ & $p(\mathrm{MPa})$ & $\rho\left(\mathrm{kg} \cdot \mathrm{m}^{-3}\right)$ & $\eta(\mathrm{mPa} \cdot \mathrm{s})$ \\
\hline \multicolumn{3}{|c|}{$T=213.45 \mathrm{~K}$} & \multicolumn{3}{|c|}{$T=298.41 \mathrm{~K}$} \\
\hline 0.11 & 941.1 & 2.723 & 0.11 & 862.3 & 0.5515 \\
\hline 0.11 & 941.1 & $2.734^{a}$ & 0.11 & 862.3 & $0.5536^{a}$ \\
\hline 1.13 & 941.6 & $2.752^{a}$ & 0.13 & 862.3 & 0.5517 \\
\hline 2.49 & 942.2 & 2.792 & 2.52 & 864.1 & 0.5620 \\
\hline 2.49 & 942.2 & $2.799^{a}$ & 2.52 & 864.1 & $0.5635^{a}$ \\
\hline 5.02 & 943.4 & 2.864 & 14.94 & 873.2 & 0.6195 \\
\hline 5.02 & 943.4 & $2.852^{a}$ & 14.94 & 873.2 & $0.6205^{a}$ \\
\hline 9.98 & 945.8 & 3.043 & 19.94 & 876.7 & 0.6411 \\
\hline 10.02 & 945.8 & 3.027 & 19.94 & 876.7 & $0.6424^{a}$ \\
\hline 10.02 & 945.8 & $3.039^{a}$ & 20.02 & 876.7 & 0.6418 \\
\hline 15.11 & 948.2 & 3.187 & & & \\
\hline 15.11 & 948.2 & $3.203^{a}$ & & & \\
\hline 15.23 & 948.2 & 3.199 & & & \\
\hline 15.23 & 948.2 & $3.201^{a}$ & & & \\
\hline 20.05 & 950.4 & $3.346^{a}$ & & & \\
\hline 20.05 & 950.4 & 3.345 & & & \\
\hline
\end{tabular}

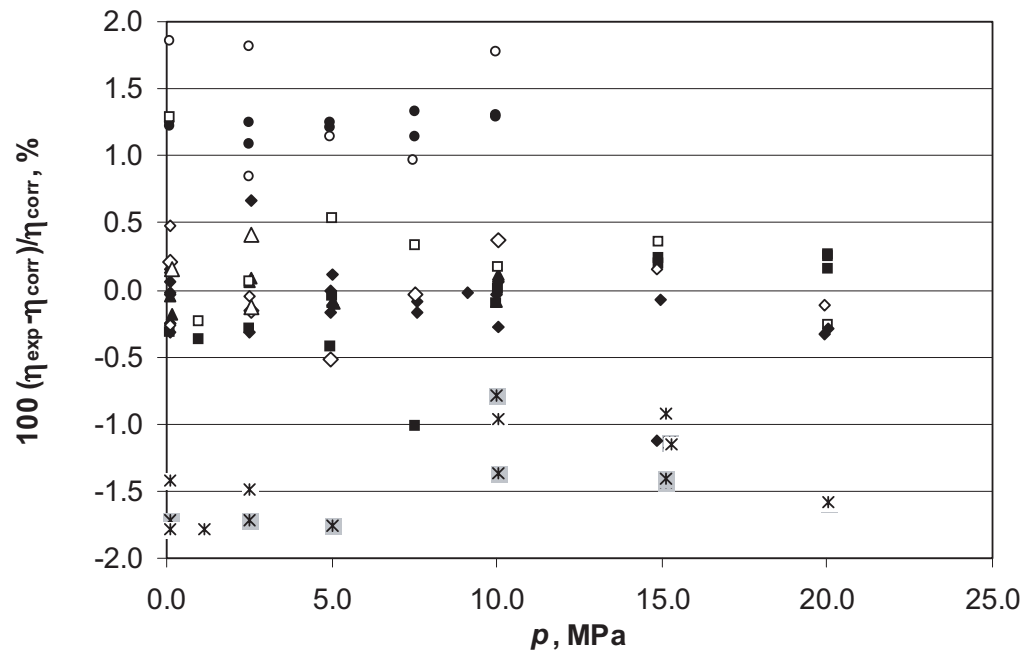

Fig. 3. Deviations of the present measurements from the correlation of Assael et al. [15]. Forced mode: $(\diamond) 298.31 \mathrm{~K},(\boldsymbol{\Delta}) 273.81 \mathrm{~K},(\bullet) 248.47 \mathrm{~K}$, (口) $222.57 \mathrm{~K}$, (困) $213.45 \mathrm{~K},(\diamond) 298.41 \mathrm{~K}$; Free decay mode: $(\diamond) 298.31 \mathrm{~K},(\triangle) 273.81 \mathrm{~K},(\bigcirc)$ $248.47 \mathrm{~K},(\square) 222.57 \mathrm{~K},(*) 213.45 \mathrm{~K},(\diamond) 298.41 \mathrm{~K}$. 
has been detected within the experimental precision of the measurements. Furthermore, it should be noted that, for the same experimental conditions, measurements were performed for both modes of operation and the values are appropriately identified in Table II.

For comparison purposes we used the viscosity correlation developed by Assael et al. [15], which has an estimated uncertainty of $\pm 2.7 \%$ within a $95 \%$ confidence limit. This correlation, based on the most recent experimental data, covers a temperature range from 213 to $373 \mathrm{~K}$ and a pressure range from atmospheric up to $250 \mathrm{MPa}$. However, at low temperatures and high densities the uncertainty in the correlation is the greatest [15] (up to $4 \%$ for density values above $920 \mathrm{~kg} \cdot \mathrm{m}^{-3}$ ) because of the scarcity of the experimental data and their dispersion. Indeed, it should be pointed out that the present work provides the first measurements below $217 \mathrm{~K}$.

The experimental values obtained by both modes of operation are compared with the above-mentioned correlation in Fig. 3. The comparison shows that the actual measurements are well within the overall uncertainty of the correlation over the entire range of conditions.

\section{CONCLUSIONS}

The results of new measurements of the viscosity of liquid toluene have been presented, which expand the available published experimental data in the very low temperature region (down to $213 \mathrm{~K}$ ) and pressures up to $20 \mathrm{MPa}$. Our results are intended to supply data for these thermodynamic regions, and thus reinforce the establishment of toluene as a standard reference fluid for viscosity.

As a final note it should be stressed that the verification of the internal consistency between the two different modes of operation in the present measurements constitutes a stringent validation of the correct operation of the present viscometer.

\section{ACKNOWLEDGMENTS}

The present work has been supported by the Fundação para a Ciência e a Tecnologia (Portugal) under PRAXIS and POCTI programmes, with European Community funds (FEDER) and national funds.

\section{REFERENCES}

1. A. A. H. Pádua, J. M. N. A. Fareleira, J. C. G. Calado, and W. A. Wakeham, Int. J. Thermophys. 17:781 (1996). 
2. A. A. H. Pádua, J. M. N. A. Fareleira, J. C. G. Calado, and W. A. Wakeham, J. Chem. Eng. Data 41:731 (1996).

3. A. A. H. Pádua, J. M. N. A. Fareleira, J. C. G. Calado, and W. A. Wakeham, J. Chem. Eng. Data 41:1488 (1996).

4. H. M. T. Avelino, J. M. N. A. Fareleira, and W. A. Wakeham, Int. J. Thermophys. 24:323 (2003).

5. J. L. Correia da Mata, J. M. N. A. Fareleira, C. M. B. P. Oliveira, F. J. P. Caetano, and W. A. Wakeham, High Temp.-High Press. 33:669 (2001).

6. J. L. Correia da Mata, J. M. N. A. Fareleira, P. M. B. S. Girão, and W. A. Wakeham, to be published.

7. T. Retsina, S. M. Richardson, and W. A. Wakeham, Appl. Sci. Res. 43:127 (1986).

8. T. Retsina, S. M. Richardson, and W. A. Wakeham, Appl. Sci. Res. 43:325 (1987).

9. A. A. H. Pádua, J. M. N. A. Fareleira, J. C. G. Calado, and W. A. Wakeham, Rev. Sci. Instrum. 69:2392 (1998).

10. C. M. B. P. Oliveira, Viscosity of Liquid Hydrocarbons at High Pressure, Ph.D. thesis (Imperial College of Science, Technology and Medicine, London, United Kingdom, 1991).

11. M. J. Assael, C. M. B. P. Oliveira, M. Papadaki, and W. A. Wakeham, Int. J. Thermophys. 13:593 (1992).

12. C. S. Barrett, Structure of Materials (McGraw-Hill, New York, 1952).

13. F. A. Gonçalves, K. Hamano, J. V. Sengers, and J. Kestin, Int. J. Thermophys. 6:641 (1987).

14. H. Kashiwagi, T. Hashimoto, Y. Tanaka, H. Kubota, and T. Makita, Int. J. Thermophys. 3:201 (1982).

15. M. J. Assael, H. M. T. Avelino, N. K. Dalaouti, J. M. N. A. Fareleira, and K. R. Harris, Int. J. Thermophys. 22:789 (2001). 